\title{
Proactive and reactive inventory policies in humanitarian operations
}

\author{
Masoud Rabbani $^{\mathbf{a}^{*} \text {, Neda Manavizadeh }}{ }^{\mathrm{b}}$, Mehran Samavati ${ }^{\mathrm{a}}$ and Moeen Sammak Jalali ${ }^{\mathrm{a}}$
}

${ }^{a}$ School of Industrial Engineering, College of Engineering, University of Tehran, Tehran, Iran ${ }^{b}$ Department of Industrial Engineering, KHATAM Institute of Higher Education, Tehran, Iran

\section{H R O N I C L E}

\begin{tabular}{l}
\hline Article history: \\
Received December 18, 2014 \\
Accepted March 162015 \\
Available online \\
March 172015 \\
\hline Keywords: \\
Humanitarian relief supply chain \\
Proactive and reactive inventory \\
policies \\
Perishable relief supplies \\
Continuous review
\end{tabular}

\section{Introduction}

The number and scale of humanitarian relief operations has considerably risen during the last decade due to the ever-increasing number of natural disasters. This has presented scholars with the imperative to conduct various research associated with emergency planning. A specific problem in the context of humanitarian operations is developing proper plans for relief inventory-related issues. In general, disaster relief lifecycle is partitioned into two separate phases: preparedness and response phase (Tufinkgi, 2006; Kovács \& Spens, 2007; Thomas \& Kopczak, 2007; Tomasini \& Van Wassenhove, 2009). Preparedness phase aims at decreasing the response time through procurement and prepositioning of relief supplies in advance of an extreme event. During the response stage, required actions are taken to support an affected community directly after the onset of a disaster. Here, a shortterm immediate reaction phase and a long-term recovery phase (i.e., reconstructing the infrastructure, retrieving the victims, and mitigating damages in the disaster zones) are differentiated. This paper focuses on inventory planning for both preparedness and response phases.

\footnotetext{
* Corresponding author

E-mail address: mrabani@ut.ac.ir (M. Rabbani)

(c) 2015 Growing Science Ltd. All rights reserved. doi: 10.5267/j.uscm.2015.3.004
}
Inventory planning for the pre- and post-disaster phases of disaster relief lifecycle is a

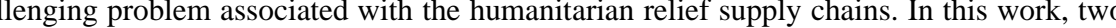 accounting for holding costs of perishable supplies, a two-stage stochastic programming model is first developed by which the inventory prepositioning locations, inventory levels, and short- term distribution quantities are determined. For the recovery phase, this research adapts the well-known continuous review $(Q, r)$ inventory model for relief warehouses while accounting case study of Iranian Red Cross is also provided to illustrate the applicability of the first model and to demonstrate how it supports the two first phases of disaster lifecycle. Additionally, a numerical example is presented to demonstrate the applicability of the $(Q, r)$ model for the recovery phase. Lastly, the impact of penalty costs on the solutions is discussed.


The main action in preparedness phase is prepositioning of relief commodities in order to decrease the immediate response time. This action comprises several key decisions needing to be implemented in pre-disaster, including the determination of storage (i.e., prepositioning) locations and stocking levels for relief items (Rawls \& Turnquist, 2012). A number of quantitative models in disaster relief literature have addressed prepositioning problems. However, some factors, such as inventory policies for perishable products and replacement-related costs, have remained unexplored. There are many sources of unpredictability in disaster relief, such as location, timing, and intensity of sudden-onset disasters (e.g. earthquakes, volcanic eruptions, and floods).

These uncertainties necessitate strategic long-term proactive/prepositioning decisions, and hence the prepositioned supplies may be remained in storage facilities for long times. Therefore, inventoryplanning policies of prepositioned supplies incorporate periodically replacement of perishable goods. Considering the long-term nature of inventory placement decisions, it comes to view that the costs associated with perishable goods (i.e., fresh goods ordering costs, old goods removing costs, disposal costs, and expected transportation costs) can have considerable effect on these decisions. However, many of the location determination models only involved factors such as characteristics of potential events expected holding cost of items, fixed opening cost of storage facilities, and proactive response time when determining the optimal prepositioning locations and inventory quantities. In this paper, prepositioning locations and stocking levels will be derived subject to the inventory policies associated with perishable goods as well as mentioned factors.

The short-term response phase is mainly related to the allocation of relief resources to the beneficiaries after a disaster strikes. During the initial hours, a large number of evacuees are arrived at shelter locations, and so the immediate provision of needed relief items is of vital importance. Since there is a little opportunity for meeting the demand from suppliers, the pre-positioned relief commodities are mainly used for distribution to the affected areas.

Short-term resource allocation decisions cannot be optimal if they are not made simultaneously with location decisions. This has led to extensive research in the area of relief prepositioning where both the preparedness and response planning are integrated by using the two stage scenario-based approaches. This work also involves the decisions related to the initial distribution of emergency items in addition to the aforementioned prepositioning decisions through a set of scenarios. The "first-stage" decisions (i.e. which are made before the characteristics of any event are known) involve the selection of optimal locations among a network of regional depots where existing warehouses are considered as candidate locations to preposition supplies, inventory quantities to be stored at each warehouse, and inventory control policy for perishable items. The "second-stage" decisions (response decisions) aim at delivering of available supplies to satisfy demands in specific areas impacted by a given event scenario, such that both expected expenses and unsatisfied demands are to be minimized.

The next steps of emergency relief occur during the recovery and reconstruction phase, where damaged properties and infrastructures are rebuilt and sheltered victims are gradually returned to their houses. According to Rath and Gutjahr (2011), this stage is considerably longer than immediate response phase, lasting usually from several weeks up to two years after a disaster. During the recovery period, the main challenge is how to manage relief activities in an unpredictable environment. Although the reconstruction phase deals with more predictable demand and possibility to plan for constant schedules compared to the shorter-time response phase, the exact timings and amounts of demands occurred during the implementation of reconstruction programs are still difficult to be recognized. These difficulties make the inventory management of relief warehouses, supporting the long-term emergency relief operations, significantly complex. The issues existing in conventional inventory control (e.g. holding and ordering costs) add to the complexity of this problem. Despite the large amount of improvements carried out in the commercial sector, there has been only a limited body of research in long-term humanitarian operations in the area of inventory control. To fulfil this gap, this research 
adapts the well-known continuous review $(Q, r)$ inventory model for relief warehouses for the longterm recovery phase while accounting for the inherent epistemic uncertainty in the required data by using an efficient possibilistic programming.

Accordingly, this paper makes the following contributions to the literature of humanitarian relief chains. Firstly, we incorporate inventory policies of perishable goods into the prepositioning planning. To the best of our knowledge, this is the first research in the preparedness domain to consider the effects of holding costs of perishable items on inventory prepositioning and short-term response distribution decisions. A dynamic two-stage stochastic programming model is proposed, which determines (1) when the old perishable items are to be replaced? (2) how much fresh supplies are to be purchased in each replenishment cycle? (3) which potential depots are to be selected for prepositioning? (4) what amount of supplies are to be prepositioned at each location? (5) which storage locations should distribute the emergency prepositioned items to an affected area?.

Secondly, a continuous review $(Q, r)$ inventory model is proposed in which the order quantities and reorder points are determined for the long-term recovery period. As far as the authors of this paper concerned, the only research that has developed a $(Q, r)$ inventory model is that of Beamon and Kotleba (2006) in which uncertainty in demands have been addressed by defining uniform distribution. They also assumed that the demand during the stock-out period is completely backorderd. Indeed, owing to the unique condition of long-term responses it is far-fetched to suggest whether stock-out demands should be backorderd or lost. According to the Consuelos Salas (2012), victims cannot probably withstand without some items, such as foods, more than two or three days. Therefore, backorderd demands should be changed to the lost ones after these days. To cope with this matter, our $(Q, r)$ model considers a mixture of backorderd and lost demands in which backorderd demands will be transformed to lost demands if the stock-out period lasts more than three days. Noteworthy, those three days can be easily modified to two, four, or any other number of days as needed. To the best of the authors' knowledge, considering both backorder and lost demands simultaneously has been developed in neither humanitarian operations nor commercial inventory control literature. For the proposed model, an effective fuzzy ranking approach based on the possibilistic mean operator is adopted to achieve the optimal solution. Finally, two sensitivity analyses on penalty costs are carried out for both models.

The remainder of the paper is organized as follows. Section 2 presents the relevant literature from the humanitarian context as well as the commercial inventory control systems. Section 3 illustrates the two proposed mathematical models. In order to illustrate the applicability of model I, a case study is provided in section 4 . Section 5 is related to the computational results and the respective sensitivity analyses. In section 5, a numerical example is presented regarding model II. The last section is related to the conclusion and future research directions.

\section{Literature review}

A large proportion of the prepositioning literature addresses location of storage facilities, stock levels of relief commodities, and immediate distribution of relief commodities. Several prepositioning models combine the post-disaster short-term response decisions with inventory placement decisions through scenario-based approaches, including two-stage stochastic programming.

This section discusses about the relevant literature classified by the decisions involved as well as the literature of perishable goods inventory policies. In addition, the existing literature regarding the inventory control models for the long-term response phase is reviewed. Lastly, a brief survey on continuous inventory control models review in the context of commercial operations is presented. 


\subsection{Relief prepositioning network}

Several authors have addressed the problem of storage locations and stocking quantities for emergency supplies. In Rawls and Turnquist (2010) approach, the problem for hurricane disasters was faced by the gulf coast of the United States. They proposed a mixed-integer stochastic programming model to determine the location and quantities of various relief commodities to be stored. Incorporating (I) the fixed cost of opening a facility; (II) variable purchase cost for commodities; (III) expected distribution cost; (IV) penalties for unmet demands, the authors consider a cost-based perspective for finding the optimal solution. Balcik and Beamon (2008) addressed this problem using a stochastic-based programming model. They formulated a maximal covering location model in which the number and location of the distribution centers as well as the amount of prepositioned relief supplies are determined aiming to maximize the total expected demand covered by the distribution centers. Salmeron and Apte (2010) consider prepositioning strategy involving location, capacity expansion, resource, and quantity determination decisions. Their main contribution was to partition the affected community into three categories: stay-back population, critical population, and transfer population. The stay-back population was the one who only requires delivery of certain items from distribution centers. The critical population includes the victims who are in emergency need of medical evacuation to relief locations. The transfer population requires temporary accommodation in relief locations. Davis et al. (2013) developed a stochastic mathematical model to determine how relief supplies should be prepositioned and distributed among a network of cooperative warehouses. The model considers traffic congestion resulting from possible evacuation behavior while incorporating time constraints for providing effective response. They apply short-term information of hurricane forecasting, and therefore their prepositioning strategy is not applicable for longer-term situations (e.g. for preparedness against earthquake).

\subsection{Short-term response}

Several researches have aimed at post-disaster immediate distribution of relief supplies. Haghani and Oh (1996) proposed a network flow model incorporating detailed load plans for distributing commodities in the aftermath of an event. Barbarosoglu and Arda (2004) extended that model to account for uncertainty in demands, capacities of network arcs, and available inventory by means of using scenarios, which is a link between pre- and post-event decisions. Several authors also integrate the stocking level and location decisions with short-term decisions. Important examples include the work of Ozbay and Ozguven (2007), Ukkusuri and Yushimito (2008), and Rawls and Turnquist (2012). In order to minimize "stock out" probability, Ozbay and Ozguven (2007) developed an inventory model to determine stocking quantities subject to uncertain demands and delivery schedule at a relief location. Ukkusuri and Yushimito (2008) addressed location determination problem aiming to minimize the probability of uncovered demand points. Rawls and Turnquist (2012) addressed the prepositioning network and short-term distribution decisions simultaneously. They assumed that the evacuees arrive at shelter locations in different times (during the first 72 hours after a disaster strikes). A mixed-integer two-stage stochastic programming model was proposed to cope with uncertainty in demands. However, similar to the other mentioned research works, they have not considered inventory policies for holding perishable goods in their relief network.

\subsection{Pre-disaster perishable inventory control}

To the best of our knowledge there is a huge research gap in the disaster operations management (DOM) literature for exploring appropriate inventory policies for perishable relief items, and despite of its importance as suggested by Altay and Green (2006), these polices have not been incorporated in the prepositioning network decisions. Whybark (2007) emphasized that the management of disaster relief inventories is an increasingly important area for scientific research in the context of humanitarian operations. He expresses some of the main differences between the enterprise (i.e., commercial) and 
disaster relief inventories. He also declares that many of the relief items such as foods and medicines are perishable, and this has several implications for their periodically management. According to him, owing to the fact that the timing of need is uncertain, the time-dependent items must be replenished with fresh supplies. Moreover, besides the well-known factors for location decisions, the storage location should be selected because the relief inventories must be always accessible as removals are scheduled. This fact is our main motivation to develop novel decision models while account for such factors.

\subsection{Inventory control of relief items in the recovery phase}

According to the Beomon and Kotleba (2006), humanitarian relief operations may even last for many years. However, mathematical modeling of Inventory management for long-term responses has received little attention in the literature. The only work in this area could be the research of Beamon and Kotleba (2006) in which a $(Q, r)$ model is proposed subject to uncertainty in demand. They cope with uncertain demand using uniform distribution, and consider only backorderd demands in their model. As it was mentioned earlier, a mixture of backorderd and lost demands needs to be studied in continuous review models in the contest of disaster relief. The only previous research considering both of demands during the stock out period has been conducted by Wang et al. (2012) in a commercial setting. They assume that a specific fraction of demand is backordered during the stock out period and the remainder is considered as lost sale, but the amount of backorder is fixed and cannot be transformed to the lost demands throughout the time.

\section{Proposed mathematical models}

\subsection{Two-stage stochastic programming model (Model-I)}

In order to improve the current relief-prepositioning network, we start with a network of regional depots where existing warehouses are considered as candidate locations. The model aims to select the best storage locations in pre-disaster by maximizing the satisfied demand in an affected area. After an extreme event strikes a particular area, the warehouse of the same area- if has already made availableand some certain warehouses in neighborhood begin deploying emergency items. Since the timing of occurrence of a disaster is unknown, the prepositioned perishable supplies should be monitored continually to guarantee that they are still healthy and useful when the need arises. This incorporates replenishment costs into the inventory prepositioning plans in addition to time and costs of transportation of items to potential demand points. Each perishable item must be removed when it is close to its expiry date, and replaced with fresh supplies. Indeed, the provision cost of each fresh item varies depending on the location of each warehouse. This variation could result from different distances from the same supplier-and hence different transportation cost- and similar factors. Furthermore, the removal of each item has its own cost. However, the old commodities can be sold in the market, and depending on the expiry date, the price of sale varies. For instance, a particular item having four months to its expiry date can be sold in a higher price in comparison with an item having one month to be spoiled. Moreover, there may be a lag between removal of old supplies and stocking fresh items, rebounding to an increase in the likelihood of shortage (if a disaster occurs during this period). Accordingly, accounting for the aforementioned replenishment expenses and lead times in prepositioning plans, which may affect the prepositioning decisions considerably, is of vital importance.

The developed mathematical model for relief prepositioning planning aims to minimize the expected costs resulting from the selection of storage locations, periodically replenishment of perishable goods, distribution of supplies to the affected areas, unmet demand penalties, and spoilage penalties. The assumptions used for model formulation are as follows: 
(1) The uncertainty in demand is addressed by defining a set of discrete scenarios.

(2) Definition of each scenario includes the forecasted demand, location of demand point, and characteristics (Which ones? likelihood...) of a specific disastrous event;

(3) Decision horizon consists of a set of time periods, $t=1,2, \ldots, T$

(4) All commodities are consumable and perishable;

(5) Each selected facility has a limited capacity;

(6) The shortages resulting from the lag of one or more periods between removal and provision are converted to costs using penalty coefficients.

\section{Notation}

Indexes

$N \quad$ Set of locations $(i \in N)$

$N \quad$ Set of affected areas $(j \in N)$

$K \quad$ Set of commodities

$T \quad$ Set of months in the planning horizon; length of planning horizon

$S \quad$ Set of scenarios

$H_{k} \quad$ Number of lifetime months of item $k$

\section{Input parameters}

$n_{k} \quad$ Unit removal cost of item $k$

$D_{j k}^{s} \quad$ Number of demands of item $k$ at demand point (affected location) $j$ in scenario $S$

$e_{i j k} \quad$ Unit shipment cost of item $k$ from location $i$ to affected location $j$

PC Unit cost for unmet demand

$c_{k} \quad$ Unit purchase cost of item $k$

$l_{k}^{a} \quad$ Unit sale cost of item $k$ with $a$ lifetime months

$E c_{k} \quad$ Unit penalty cost for shortage of item $k$ between the removal of old commodities and provision of fresh supplies

$c c_{i} \quad$ Capacity of storage facility in location $i$

$p_{j}^{s} \quad$ Occurrence probability of scenario $s$ at location $j$

$f_{k i} \quad$ Unit provision cost of commodity $k$ for the storage facility of location $i$

\section{Decision variables}

$x_{i j k}^{s} \quad$ Number of commodities $k$ shipped from location $i$ to affected area $j$ for scenario $s$

$r_{k i} \quad$ Number of commodities $k$ prepositioned at location $i$ ( a first-stage decision variable)

$b_{k i t}^{a} \quad$ Number of commodities $k$, with $a$ lifetime months, removed from the storage facility of location $i$ in period $t$ ( a first-stage decision variable) 
$u_{j k}^{s} \quad$ Number of unmet demands of commodity $k$ at affected area $j$ for scenario $s$

$Q_{\text {kit }} \quad$ Number of purchased commodities $k$ for the storage facility of location $i$ in period $t$ (a first-stage decision variable)

$E_{\text {kit }} \quad$ Shortage of item $k$ due to the lag between the removal of old commodities and provision of fresh supplies ( a first-stage decision variable)

$I_{\text {kit }}^{a} \quad$ Inventory level of item $k$ with a lifetime months at location $i$ in period $t$ ( a first-stage decision variable)

\section{Model}

$$
\begin{aligned}
& \min \sum_{s}\left[\sum_{i} \sum_{j} \sum_{k} p_{j}^{s}\left(e_{i j k} x_{i j k}^{s}\right)+\sum_{j} \sum_{k} p_{j}^{s} u_{j k}^{s} P C\right]+\sum_{k} \sum_{i} \sum_{t=1}^{T} \sum_{a=0}^{H_{k}} n_{k} b_{k i t}^{a}+ \\
& \sum_{t} \sum_{k} \sum_{i} c_{k} Q_{k i t}+\sum_{k} \sum_{i} \sum_{t} f_{k i} Q_{k i t}-\sum_{k} \sum_{i} \sum_{t} \sum_{a}^{H_{k}} L_{k}^{a} b_{k i t}^{a}+\sum_{k} \sum_{i} \sum_{t} E C_{k} E_{k i t}
\end{aligned}
$$

subject to

$b_{k i t}^{a} \leq I_{k i t}^{a} \quad \forall t \in T, k \in K, a \in H_{k}, i \in N$

$I_{k i t+1}^{a-1}=I_{k i t}^{a}-b_{k i t}^{a} \quad \forall t \in T, k \in K, a \geq 1, i \in N$

$I_{\text {kit }}^{H_{k}}=Q_{k i t} \quad \forall t \geq 2, k \in K, i \in N$

$I_{k i 1}^{H_{k}}=r_{k i} \quad \forall k \in K, i \in N$

$E_{k i t}=r_{k i}-\sum_{a=1}^{H_{a}} I_{k i t}^{a} \quad \forall k \in K, i \in N, t \in T$

$\sum_{a=0}^{H_{k}} I_{k i t}^{a} \leq r_{k i} \quad \forall k \in K, i \in N, t \in T$

$\sum_{k=1}^{k} r_{k i} \leq C c_{i} \quad \forall i \in N$

$u_{j k}^{s}=D_{j k}^{s}-\sum_{i \neq j} x_{i j k}^{s} \quad \forall k \in K, j \in N, s \in S$

$\sum_{j \in N} x_{i j k}^{s} \leq r_{k i} \quad \forall k \in K, i \in N, s \in S$

$x_{i j k}^{s}, r_{k i}, b_{k i t}^{a}, u_{j k}^{s}, Q_{k i t}, f_{k i}, E_{k i t} \geq 0 \quad \forall t \in T, k \in K, a \in H_{k}, i, j \in N, s \in S$

Constraint (2) ensures that the number of supplies removed from location $i$ does not exceed the inventory level of the same supply. Eq. (3) is called balance constraint that plays the role of the memory for available supplies in each period. In other words, this constraint states that the stocking level of item $k$, with $a-1$ remaining lifetime months, in location $i$ in period $t+1$ equals that of item $k$ with $a$ remaining lifetime months in period $t$ minus the number of item $k$ removed out in period $t$. Constraint (4) guarantees that the inventory level of fresh supplies is equal to the purchased items in each period. Eq. (5) ensures that inventory level of each item in each location equals the prepositioning level of that 
item in the first period. Constraint (6) specifies the shortage caused by the lag between removal and provision. Eq. (7) ensures that $r_{k i}$ must not be exceeded by stocking level in any period, as it is the optimal prepositioning level. Constraint (8) is related to the capacity limits associated with facilities. Eq. (9) computes the amount of unmet demand for each scenario. The last constraint states that the shipment amount is less than the prepositioning level.

\subsection{Long-term response mathematical model (Model-II)}

There are two separate times for acquisition of emergency items. The first one is the pre-disaster acquisition and storage of these items in anticipation of a disaster. The second one is the development of sources for acquisition of items for delivery during a certain relief operation. In this section, a fuzzy continuous review inventory model is proposed in which the imprecise demand parameters are considered as possibility distributions in the form of fuzzy triangular numbers whose prominent points have been estimated based upon the available objective data and expert's subjective opinions. The model aims to find the order quantity as well as the re-order point of each relief item by minimizing the relevant costs and possible shortages during the long-term relief operations.

The following notations are used to formulate the long-term relief inventory control model.

$\widetilde{D} \quad$ Demand for the whole recovery period, a triangular fuzzy number

$\tilde{d} \quad$ Demand during lead time, a triangular fuzzy number

$r \quad$ Reorder point

Q $\quad$ Order quantity

A Fixed cost of placing an order

$\pi \quad$ Shortage cost of lost demand, per unit

p Shortage cost of backorder demand, per unit

$h \quad$ Unit inventory holding cost, for the whole planning horizon

$\tau \quad$ Lead time

\section{Definition 1}

According to Carlsson \& Fullér (2001), for a given fuzzy number $\widetilde{A}$, the possibilistic mean value is defined as:

$$
\bar{M}(\widetilde{A})=\int_{0}^{1} \alpha\left(\mathrm{A}_{\alpha}^{-}+\mathrm{A}_{\alpha}^{+}\right) \mathrm{d} \alpha
$$

where interval $\left[\mathrm{A}_{\alpha}^{-}, \mathrm{A}_{\alpha}^{+}\right]$denotes the alpha-cut set $\mathrm{A}_{\alpha}$. Accordingly, the total expected cost is formulated as follows:

$$
\begin{aligned}
& \widetilde{C}(Q, r)=\left(\frac{\widetilde{D}}{Q}\right) A+h\left(\frac{Q}{2}-\frac{3 \lambda}{2}+\bar{M}(\widetilde{d}-r)^{-}\right)+\pi\left(\bar{M}(\widetilde{d}-r)^{+}-3 \lambda\right) \frac{\widetilde{D}}{Q}+3 p \lambda \frac{\widetilde{D}}{Q} \quad T \geq 3 \\
& \widetilde{C}(Q, r)=\left(\frac{\widetilde{D}}{Q}\right) A+h\left(\frac{Q}{2}-\frac{T \lambda}{2}+\bar{M}(\widetilde{d}-r)^{-}\right)+T p \lambda \frac{\widetilde{D}}{Q} \quad T \prec 3
\end{aligned}
$$


The first expression is related to the situation in which backorder demands in a cycle are more than three days, and so the demands of the last three days of the cycle can only be met. The rest of the demands is considered as lost demand which never get satisfied. In the second expression, the stockout period is shorter than three days, and therefore they all can be considered as backorder demand. Furthermore, $T$ and $\lambda$ are defined as follows (see Figure 1).

$\lambda=\frac{\bar{M}(\widetilde{d})^{\tilde{d} \succ r}}{\tau}, \bar{M}(\widetilde{d})^{\tilde{d} \succ r}$ denote the possibilistic mean value of $\widetilde{d}$ such that shortage will occur during lead time, $\tau$ and $T=\frac{\bar{M}(\widetilde{d}-r)^{+}}{\lambda}$.

In addition, $\bar{M}(\widetilde{d}-r)^{+}$is possibilistic mean of probable shortage during a replenishment cycle; In other words, this is the expected amount of shortage that will probably occur during a cycle. On the other hand, $\bar{M}(\widetilde{d}-r)^{-}$is the expected amount of $r-\tilde{d}$ when $r-\tilde{d} \geq 0$.

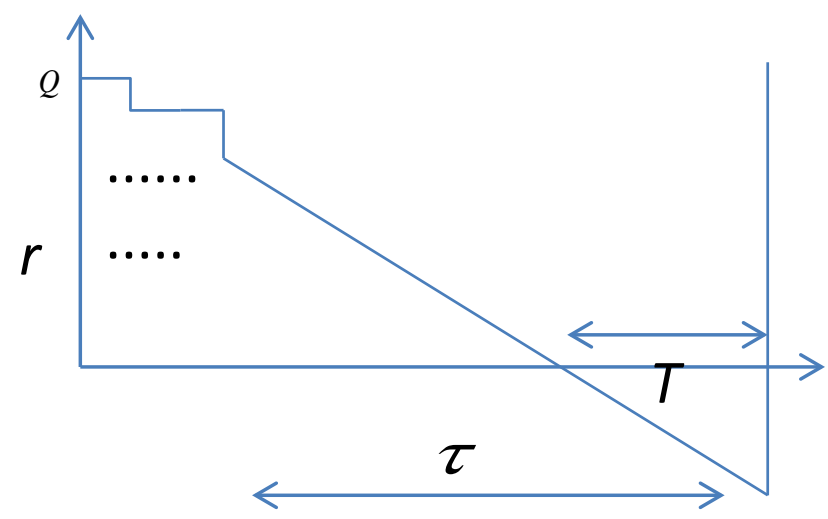

Fig. 1. A schematic view of a single cycle

\subsection{Analysis of model II}

In this section, the value of $\bar{M}(\widetilde{d}-r)^{-}, \bar{M}(\widetilde{d}-r)^{+}$, and $\bar{M}(\widetilde{d})^{\tilde{d} \succ r}$ will be discussed. As it is well known, the safety stock can be defined as the difference between reorder level $r$ and the expected demand $\bar{M}(\widetilde{d})$. If $r \succ \bar{d}$, there will be extra inventory-and hence extra holding cost. Moreover, the minimum level of safety stock is zero, implying $r \geq \bar{M}(\widetilde{d})$. For a given $r \operatorname{in}[\bar{M}(\widetilde{d}), \bar{d}]$, the value of $\bar{M}(\widetilde{d}-r)^{-}$, $\bar{M}(\widetilde{d}-r)^{+}$, and $\bar{M}(\widetilde{d})^{\tilde{d} \succ r}$ can be found as follows (Dutta et al. (2007)):

Situation 1. ( $\underline{d} \leq r \leq d)$, alpha level set of lead-time demand, when the demand is less than $r$, can be obtained by (see Figure 2)

$$
(\widetilde{d})_{\alpha}^{\widetilde{d} \prec r}=\left\{\begin{array}{l}
{\left[d_{\alpha}^{-}, r\right] \text { if } \mu_{\widetilde{d}}(r) \geq \alpha} \\
\varnothing \quad \text { if } \mu_{\tilde{d}}(r) \prec \alpha
\end{array}\right.
$$

which implies:

$$
(\widetilde{d}-r)_{\alpha}{ }^{-}=\left\{\begin{array}{c}
{\left[d_{\alpha}^{-}-r, 0\right] \text { if } \mu_{\tilde{d}}(r) \geq \alpha} \\
\varnothing \quad \text { if } \mu_{\widetilde{d}}(r) \prec \alpha
\end{array}\right.
$$

Referring to definition 1, we would have: 


$$
\bar{M}(\widetilde{d}-r)^{-}=\frac{\mu(r)^{3}}{3}(d-\underline{d})+\frac{\mu(r)^{2}}{2}(\underline{d})-\frac{\mu(r)^{2}}{2}(r)
$$

where $\mu(r)=\left(\frac{r-\underline{d}}{d-\underline{d}}\right)$
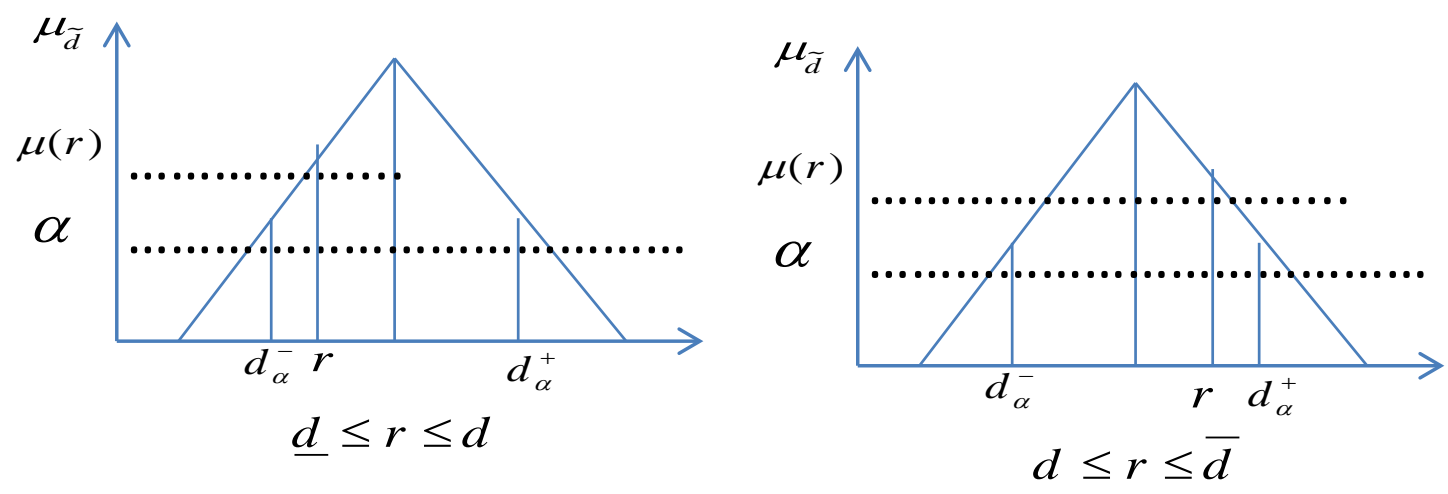

Fig. 2. Alpha cut of $\widetilde{d}$

Similarly, alpha level set of lead-time demand, when the demand is greater than $r$, can be obtained by:

$$
(\widetilde{d})_{\alpha}^{\tilde{d} \geq r}=\left\{\begin{array}{l}
{\left[r, d_{\alpha}^{+}\right] \text {if } \mu_{\tilde{d}}(r) \geq \alpha} \\
{\left[d_{\alpha}^{-}, d_{\alpha}^{+}\right] \text {if } \mu_{\tilde{d}}(r) \prec \alpha}
\end{array}\right.
$$

which implies:

$$
(\widetilde{d}-r)_{\alpha}^{+}=\left\{\begin{array}{c}
{\left[0, d_{\alpha}^{+}-r\right] \text { if } \mu_{\tilde{d}}(r) \geq \alpha} \\
{\left[d_{\alpha}^{-}-r, d_{\alpha}^{+}-r\right] \text { if } \mu_{\tilde{d}}(r) \prec \alpha}
\end{array}\right.
$$

Referring to definition 1, we would have:

$$
\bar{M}(\widetilde{d}-r)^{+}=\frac{\bar{d}}{6}+\frac{2 d}{3}+\frac{\underline{d}}{6}-\mu(r)^{2}\left[\frac{r-\underline{d}}{3}+\frac{\underline{d}}{2}\right]+\left[\frac{\mu(r)^{2}}{2} r-r\right]
$$

Also, $\bar{M}(\widetilde{d})^{\tilde{d} \succ r}$ can be obtained as follows:

$$
\bar{M}(\widetilde{d})^{\tilde{d}>r}=\frac{\bar{d}+4 d+\underline{d}}{6}-\mu(r)^{3}(d-\underline{d})-\frac{\mu(r)^{2}}{2} \underline{d}+\frac{\mu(r)^{2}}{2} r
$$

Situation 2. $(d \leq r \leq \bar{d})$, The process of obtaining the possibilistic mean values is similar to situation 1 , and finally we would have:

$$
\begin{gathered}
\bar{M}(\widetilde{d}-r)^{-}=\frac{\bar{d}}{6}+\frac{2 d}{3}+\frac{\underline{\underline{d}}}{6}-\frac{\mu(r)^{2}}{2} \bar{d}+\frac{\mu(r)^{3}}{3}(\bar{d}-d)+\left[\frac{\mu(r)^{2}}{2} r-r\right] \\
\bar{M}(\widetilde{d}-r)^{+}=-\frac{\mu(r)^{3}}{3}(\bar{d}-d)+\frac{\mu(r)^{2}}{2} \bar{d}-\frac{\mu(r)^{2}}{2}(r)
\end{gathered}
$$




$$
\bar{M}(\widetilde{d})^{\tilde{d} \succ r}=-\frac{\mu(r)^{3}}{3}(\bar{d}-d)+\frac{\mu(r)^{2}}{2} \bar{d}+\frac{\mu(r)^{2}}{2} r
$$

where $\mu(r)=\frac{(\bar{d}-r)}{(\bar{d}-d)}$.

\subsection{Defuzzification and solution of model II}

To find the optimal value of $Q$ and $r$, the possibilistic mean of expected total cost, $\widetilde{C}(Q, r)$, is required, which is:

$$
\begin{aligned}
& \bar{M}(\widetilde{C}(Q, r))=\left(\frac{A}{Q}\right)\left(\frac{\underline{D}+4 D+\bar{D}}{6}\right)+h\left(\frac{Q}{2}-\frac{3 \lambda}{2}+\bar{M}(\tilde{d-r})^{-}\right) \\
& +\pi\left(\bar{M}(d-r)^{+}-3 \lambda\right) \frac{1}{Q}\left(\frac{\underline{D}+4 D+\bar{D}}{6}\right)+3 p \lambda \frac{1}{Q}\left(\frac{\underline{D}+4 D+\bar{D}}{6}\right) \quad T \geq 3 \\
& \widetilde{M}(C(Q, r))=\left(\frac{A}{Q}\right)\left(\frac{\underline{D}+4 D+\bar{D}}{6}\right)+h\left(\frac{Q}{2}-\frac{T \lambda}{2}+\bar{M}(d-r)^{-}\right) \\
& +T p \lambda \frac{1}{Q}\left(\frac{\underline{D}+4 D+\bar{D}}{6}\right) \quad T \prec 3
\end{aligned}
$$

\subsubsection{Situation 1}

To solve $Q^{*}$ and $r^{*}$ when $(\underline{d} \leq r \leq d)$, the simultaneous equations can be obtained as follows;

By equating $\frac{\delta \bar{M}(\widetilde{C}(Q, r))}{\delta Q}=0$ for $T \geq 3$, we will obtain:

$$
Q=\sqrt{\frac{2 F\left(A+\pi\left(\bar{M}(\tilde{d-r})^{+}-3 \lambda\right)+3 p \lambda\right.}{h}}
$$

where $\mathrm{F}=\left(\frac{\underline{D}+4 D+\bar{D}}{6}\right)$ and, by equating $\frac{\delta \bar{M}(\widetilde{C}(Q, r))}{\delta r}=0$ for $T \geq 3$, we get

$$
Q=\frac{F\left[\pi\left(\frac{r-\underline{d}}{d-\underline{d}}\right)\left(\frac{2+d-\underline{d}-2 r}{2(d-\underline{d})}-\frac{3}{\tau}\right)-\pi+\frac{3 p}{\tau}\left(\frac{r-\underline{d}}{d-\underline{d}}\right)\right]}{-\frac{3 h}{2 \tau}\left(\frac{r-\underline{d}}{d-\underline{d}}\right)-\frac{h}{2}\left(\frac{r-\underline{d}}{d-\underline{d}}\right)^{2}}
$$

By equating Eq. (22) with Eq. (23), $r^{*}$ will be found. Then, $Q^{*}$ can be obtained by substituting $r^{*}$ into Eq. (23).

In addition, by equating $\frac{\delta \bar{M}(\widetilde{C}(Q, r))}{\delta Q}=0$ for $T \prec 3$, we get:

$$
Q=\sqrt{\frac{2(A F+p T F \lambda)}{h}}
$$


and, by equating $\frac{\delta \bar{M}(\widetilde{C}(Q, r))}{\delta r}=0$ for $T \prec 3$, we get:

$$
Q=\frac{p F\left[\frac{\delta T}{\delta r} \lambda+\frac{\delta \lambda}{\delta r} T\right]}{\frac{h}{2}\left(\frac{\delta T}{\delta r} \lambda+\frac{\delta \lambda}{\delta r} T\right)-h \frac{\delta \bar{M}(d-r)^{-}}{\delta r}}
$$

where:

$$
\begin{gathered}
\frac{\delta T}{\delta r}=\left[\left(\frac{r-\underline{d}}{d-\underline{d}}\right)\left(\frac{1-r}{d-\underline{d}}+\frac{1}{2}\right)-1\right] \lambda-\left[\bar{M}(\tilde{d}-r)^{+} \frac{1}{\tau}\left(\frac{r-\underline{d}}{d-\underline{d}}\right)\right] \\
\frac{\delta \lambda}{\delta r}=\frac{1}{\tau}\left(\frac{r-\underline{d}}{d-\underline{d}}\right) \\
\frac{\delta \bar{M}(\widetilde{d}-r)^{-}}{\delta r}=-\frac{1}{2}\left(\frac{r-\underline{d}}{d-\underline{d}}\right)^{2}
\end{gathered}
$$

Therefore, $r^{*}$ can be solved by equating Eq. (24) with Eq. (25). The optimum $Q^{*}$ will be found by substituting $r^{*}$ into Eq. (25).

\subsubsection{Situation 2}

To solve $Q^{*}$ and $r^{*}$ when $(d \leq r \leq \bar{d})$, the simultaneous equations can be obtained as follows; Equating $\frac{\delta \bar{M}(\widetilde{C}(Q, r))}{\delta Q}=0$ for $T \geq 3$, we have

$$
Q=\sqrt{\frac{\left(A F-\pi F\left(\bar{M}(\widetilde{d-r})^{+}-3 \lambda\right)-3 p \lambda F\right) 2}{h}}
$$

Equating $\frac{\delta \bar{M}(\widetilde{C}(Q, r))}{\delta r}=0$ for $T \geq 3$, we get:

$$
Q=\frac{p F\left[\frac{\delta T}{\delta r} \lambda+\frac{\delta \lambda}{\delta r} T\right]}{\frac{h}{2}\left(\frac{\delta T}{\delta r} \lambda+\frac{\delta \lambda}{\delta r} T\right)-h \frac{\delta \bar{M}(d-r)^{-}}{\delta r}}
$$

where:

$$
\frac{\delta T}{\delta r}=\frac{\frac{\delta \bar{M}(\widetilde{d-r})^{+}}{\delta r} \lambda-\frac{\delta \lambda}{\delta r} \bar{M}(\widetilde{d-r})^{+}}{\lambda^{2}}
$$




$$
\begin{aligned}
& \frac{\delta \lambda}{\delta r}=\frac{-\left(\frac{\bar{d}-r}{\bar{d}-d}\right)\left(\frac{r}{\bar{d}-d}\right)+\frac{3}{2}\left(\frac{\bar{d}-r}{\bar{d}-d}\right)^{2}-\left(\frac{\bar{d}-r}{\bar{d}-d}\right)\left(\frac{\bar{d}}{\bar{d}-d}\right)}{\tau} \\
& \frac{\delta \bar{M}(\overline{d-r})^{-}}{\delta r}=\left(\frac{\bar{d}-r}{\bar{d}-d}\right)\left(\frac{\bar{d}}{\bar{d}-d}\right)-\frac{1}{2}\left(\frac{\bar{d}-r}{\bar{d}-d}\right)^{2}-1-\left(\frac{\bar{d}-r}{\bar{d}-d}\right)\left(\frac{r}{\bar{d}-d}\right)
\end{aligned}
$$

By equating Eq. (33) with Eq. (34) $r^{*}$ will be found. Then, $Q^{*}$ can be solved by substituting $r^{*}$ into Eq. (6).

Moreover, Equating $\frac{\delta \bar{M}(\widetilde{C}(Q, r))}{\delta Q}=0$ for $T \prec 3$, we get:

$$
Q=\sqrt{\frac{2(A F+p T F \lambda)}{h}}
$$

and, by equating $\frac{\delta \bar{M}(\widetilde{C}(Q, r))}{\delta r}=0$ for $T \prec 3$, we obtain:

$$
Q=\frac{p F\left[\frac{\delta T}{\delta r} \lambda+\frac{\delta \lambda}{\delta r} T\right]}{\frac{h}{2}\left(\frac{\delta T}{\delta r} \lambda+\frac{\delta \lambda}{\delta r} T\right)-h \frac{\delta \bar{M}(d-r)^{-}}{\delta r}}
$$

Similarly, $r^{*}$ can be solved by equating Eq. (38) with Eq. (39) and optimum $Q^{*}$ will be found by substituting $r^{*}$ into Eq. (39).

\subsection{Sufficient condition}

In all of the four situations, the sufficient condition and range of both $r$ and $T$ must be satisfied simultaneously. In other words, the convexity of $\bar{M}(\widetilde{C}(Q, r))$ must be demonstrated in order to attain optimal $r^{*}$ and $Q^{*}$, meaning that Hessian matrix of $\bar{M}(\widetilde{C}(Q, r))$ must be positive at $\left(Q^{*}, r^{*}\right)$. That means:

$$
\begin{aligned}
& \frac{\partial^{2} \bar{M}(\widetilde{C}(Q, r))}{\partial Q^{2}} \succ 0, \\
& \frac{\partial^{2} \bar{M}(\widetilde{C}(Q, r))}{\partial Q^{2}} \frac{\partial^{2} \bar{M}(\widetilde{C}(Q, r))}{\partial r^{2}}-\frac{\partial^{2} \bar{M}(\widetilde{C}(Q, r))}{\partial Q \partial r} \succ 0 .
\end{aligned}
$$

It is obvious that $\frac{\partial^{2} \bar{M}(\widetilde{C}(Q, r))}{\partial Q^{2}}$ is always positive, and so the only sufficient condition is

$$
\frac{\partial^{2} \bar{M}(\widetilde{C}(Q, r))}{\partial Q^{2}} \frac{\partial^{2} \bar{M}(\widetilde{C}(Q, r))}{\partial r^{2}}-\frac{\partial^{2} \bar{M}(\widetilde{C}(Q, r))}{\partial Q \partial r} \succ 0
$$




\section{Case study}

As an illustration of the application of model I describing prepositioning and immediate distribution decisions, an example case has been developed. The example case is related to the current activities of "Iranian Red Crescent Society", which is one of the main humanitarian relief organization in Iran. Noteworthy, although some of the information provided here has been obtained through an interview with the organization's experts, the example case is not a real one and it is not claimed that the organization is operating in the same way.

\subsection{Background}

Iranian Red Crescent Society (IRCS) is a humanitarian relief organization undertaking the pre-and post disaster relief operations. Earthquake is the most frequently extreme event in Iran causing many fatalities each year. To cope with this situation, IRCS has considered storage facilities in each county throughout the country, for prepositioning purposes. It should be noted that according to the experts' knowledge, all of the counties, except one, have the potential to be struck by earthquake disaster. Owing to the fact that storage capacity of each single warehouse is limited, the beneficiaries of a certain affected area may not be fully satisfied by the warehouse of related county. As a result, the distribution of items from other counties' warehouses is required. The perishable supplies can be prepositioned for some limited times, and get replenished periodically. Specifically, there are 30 counties in Iran which have earthquake potential, each of which with a main warehouse controlled by IRCS organization, and we consider all of them in the example. The map in Fig. 3 shows the location of these 30 warehouses.

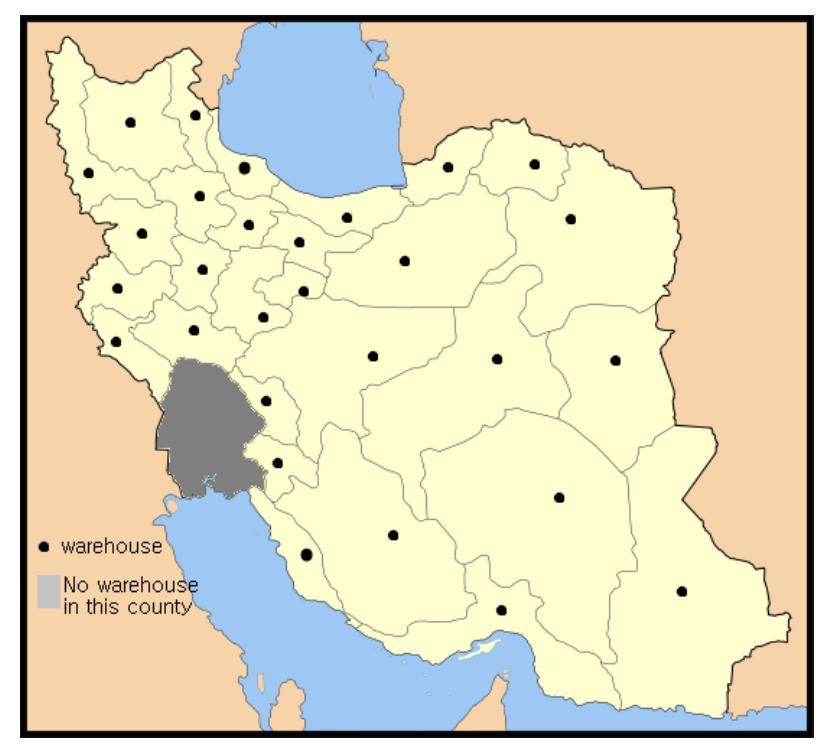

Fig. 3. Prepositioning locations across Iran

\subsection{Data characteristics}

The locations (counties) include storage facilities with capacities between 4000 and 8000 units. It is assumed that each person's relief needs requires one unit of warehouse space. We assume that there are two types of perishable commodities, with 6 and 10 months lifetimes. The planning horizon is assumed 18 months. Based on Richter scale, there are three potential earthquake scenarios, fewer than 5.5, between 5.5 and 6.5, and more than 6.5 Richter. The resulting demands of each scenario as well as their probabilities are summarized in Appendix. It is assumed that the transportation cost for moving material is \$.02 per unit-kilometer for both of the commodities (per person's material). The purchase price of each person's worth of items 1 and 2 are $\$ 100$ and $\$ 200$, respectively. Across the location of warehouses, the provision cost of both items ranges from $\$ 5$ to $\$ 50$. The removal of old items costs $\$ 0.5$ and \$1 for items 1and 2 respectively. Unit penalty cost for shortage of items between removal of 
old commodities and provision of fresh supplies is assumed to be $\$ 50$ per period. The unit sale price of old commodities varies depending on the left lifetime; the more lifetime days, the more sale price.

\subsection{Computational results and sensitivity analysis}

In this section, we present computational results of the example case study and analyze the behavior of the proposed model under different penalty cost (pc) values. The results reported below were obtained by using Lingo 8.0 optimization software. As a first experiment, we assume that the unit penalty cost of unmet demand (pc) is $\$ 150$ (the same as purchase price of item1). With $E c_{k}=\$ 150$, sixteen storage facilities are activated in which a total amount of 40025 units of item 1 and 47058 units of item 2 are stored. The total cost (excluding the penalty costs) is $\$ 908425$ including the replenishment cost of $\$ 325480$. This demonstrates the considerable effect of perishable inventories holding costs on the total cost. One of the main problems for calculations is determining appropriate values for shortage penalties. In model I, similar to the many other models in the literature, the objective function is based on cost, and so for trade-off between cost and unmet demand, it is necessary to determine an apt cost-based value for unmet demand penalties. Therefore, we ran the model for eleven times under different penalty cost values, and the results were demonstrated as a graph in Figure 4. In the figure, the values of unmet demand and total cost (excluding penalty cost $(p c)$ ) were normalized, and the penalty cost values (on the horizontal axis) initiated from zero and continued up to ten times of the purchase cost of commodity 1. It should be noted that the results are only presented for commodity 1 . According to the graph, the unmet demand values in penalty cost 0,30 , and 60 are 1, showing no demand has been satisfied. As a result, the total costs are equal to zero in this penalty costs. On the contrary, in penalty costs 270 and 300 the unmet demand values are equal to zero and total costs are at the highest value. Moreover, total cost value increases severely from penalty cost 180 onwards, whereas the unmet demand values do not decrease in the same severe trend. Consequently, the value 180 for penalty cost could be the best selection for this example. Therefore, it can be concluded that the unsatisfied demand penalty cost parameter should not either be chosen too large, as in that case, there is no additional positive effect with respect to unmet demand, while total cost still increases, or too small, as in that case the model tries to keep the demands unsatisfied. In terms of $E c_{k}$, it should be noted that its determination is difficult as it indirectly affects the value of the objective function. However, it has the same behavior as parameter $p c$ when changing its values.

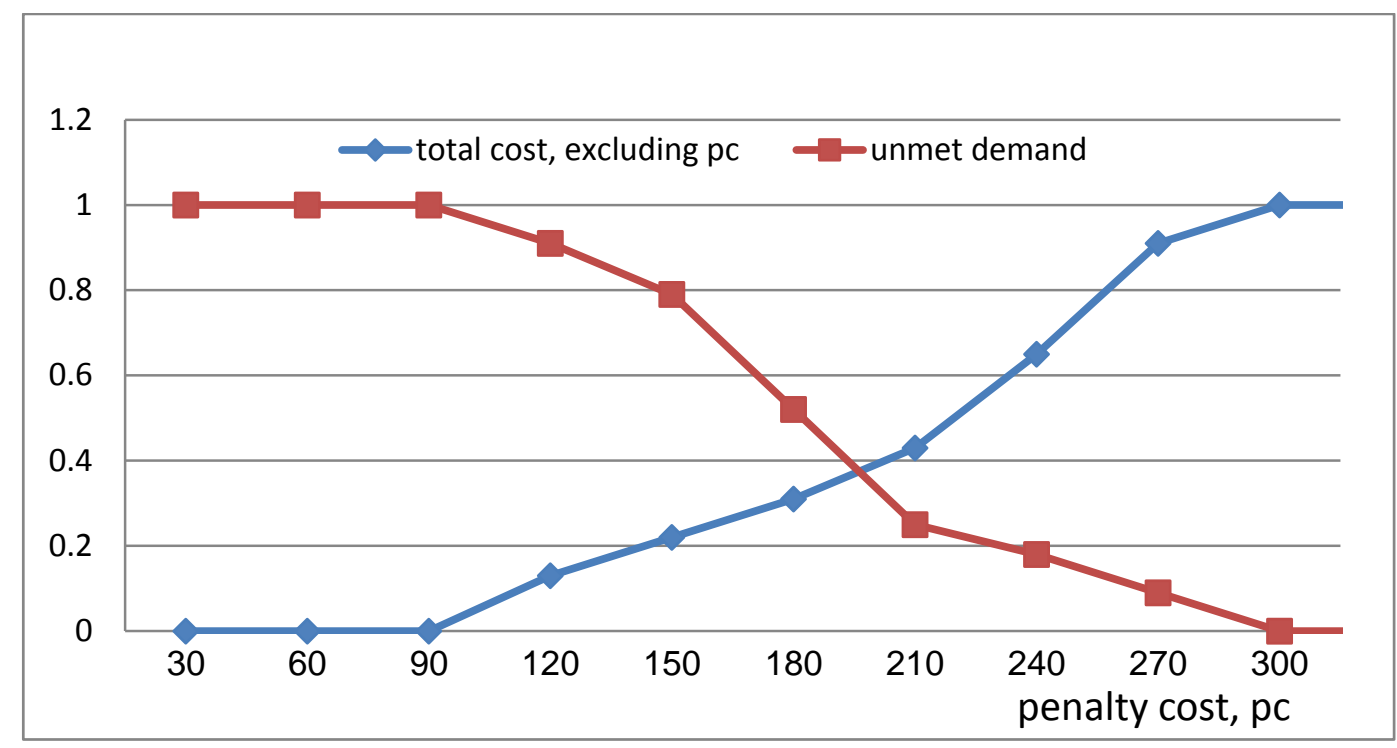

Fig. 4. Sensitivity analysis of unmet demand and total cost

In other words, if this parameter has a too small value, the replenishment processes will not be executed, and while warehouses apparently have supplies for distribution, as $r_{k i}>0$ (see constraint (10)), there 
are no enough commodities in them. In addition, if $E c_{k}$ has a too large value, it may counteract the effect of parameter $p c$-and hence no demand may be satisfied. Therefore, the best way for this parameter seems to be almost equal to $p c$.

\section{Table 1}

Analysis of the effect of holding periods

\begin{tabular}{|c|c|c|c|c|c|c|c|c|}
\hline 之 & $\begin{array}{l}\text { Problem } \\
\mathrm{I} \times \mathrm{K} \times \mathrm{t}\end{array}$ & 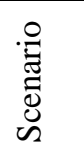 & Probability & Costs & Demands & $\begin{array}{l}\text { Total } \\
\text { costs }\end{array}$ & $\begin{array}{l}\text { Post- } \\
\text { disaster } \\
\text { costs }\end{array}$ & $\begin{array}{l}\text { Holding } \\
\text { costs }\end{array}$ \\
\hline 1 & $2 \times 2 \times 2$ & $\begin{array}{l}1,2, \\
3,4\end{array}$ & $\begin{array}{c}0.4,0.2 \\
0.3,0.1\end{array}$ & $\begin{array}{c}\text { Unif [50- } \\
\text { 2000] }\end{array}$ & Unif [1000-5000] & 15293 & 5125 & 10168 \\
\hline 2 & $4 \times 2 \times 2$ & $\begin{array}{l}1,2, \\
3,4\end{array}$ & $\begin{array}{c}0.0,0.2 \\
0.3,0.1\end{array}$ & $\begin{array}{c}\text { Unif [50- } \\
\text { 2000] }\end{array}$ & Unif [1000-5000] & 18341 & 5125 & 10992 \\
\hline 3 & $8 \times 2 \times 2$ & $\begin{array}{l}1,2, \\
3,4\end{array}$ & $\begin{array}{c}0.4,0.2 \\
0.3,0.1\end{array}$ & $\begin{array}{c}\text { Unif [50- } \\
\text { 2000] }\end{array}$ & Unif [1000-5000] & 42415 & 5125 & 2311 \\
\hline 4 & $16 \times 2 \times 2$ & $\begin{array}{l}1,2, \\
3,4\end{array}$ & $\begin{array}{c}0.4,0.2 \\
0.3,0.1\end{array}$ & $\begin{array}{c}\text { Unif [50- } \\
\text { 2000] }\end{array}$ & Unif [1000-5000] & 135240 & 5125 & 86824 \\
\hline 5 & $32 \times 2 \times 2$ & $\begin{array}{l}1,2, \\
3,4\end{array}$ & $\begin{array}{c}0.4,0.2 \\
0.3,0.1\end{array}$ & $\begin{array}{c}\text { Unif [50- } \\
\text { 2000] }\end{array}$ & Unif [1000-5000] & 483512 & 5125 & 360263 \\
\hline
\end{tabular}

\section{Analysis of the effect of perishable supplies replacement costs}

One reason for incorporating perishable supplies replacement costs into the relief warehouses location problem (model I) is their substantial impact on the location of such warehouses. In order to illustrate such effect, five problems, with different numbers of periods, $t$, are considered, where demands and costs are random parameters with uniform distribution Unif $(1000,5000)$ and Unif $(50,2000)$, respectively. For each problem, also, there are four scenarios. According to the Table 1, perishable supplies holding costs constitute a greater proportion of the total cost- compared to the post-disaster costs- and these costs obviously increase as the number of holding periods, $t$ rises. In addition, due to existing uncertainty in the timing of disasters, the number of holding periods can be considerable, and thus, perishable supplies holding costs will significantly increase. In Table 2, five other problems are considered, where $t$ is fixed, but the number of candidate storage locations, $i$, is increasing. According to the results, the total cost rises by increasing $i$, but it is not as dramatic as the $t$-related results. To simplify the comparison, the results are drawn in a graph (see figure 5). The graph shows that the figure related to parameter $t$ witnesses a dramatic rise, while the figure for $i$, in spite of having an upward trend, does not increase as significant as that. Therefore, we would reach to the conclusion that the influence of holding costs is much more than post-disaster costs, and this presents the scholars with the imperative to involve such costs in relief location-allocation problems.

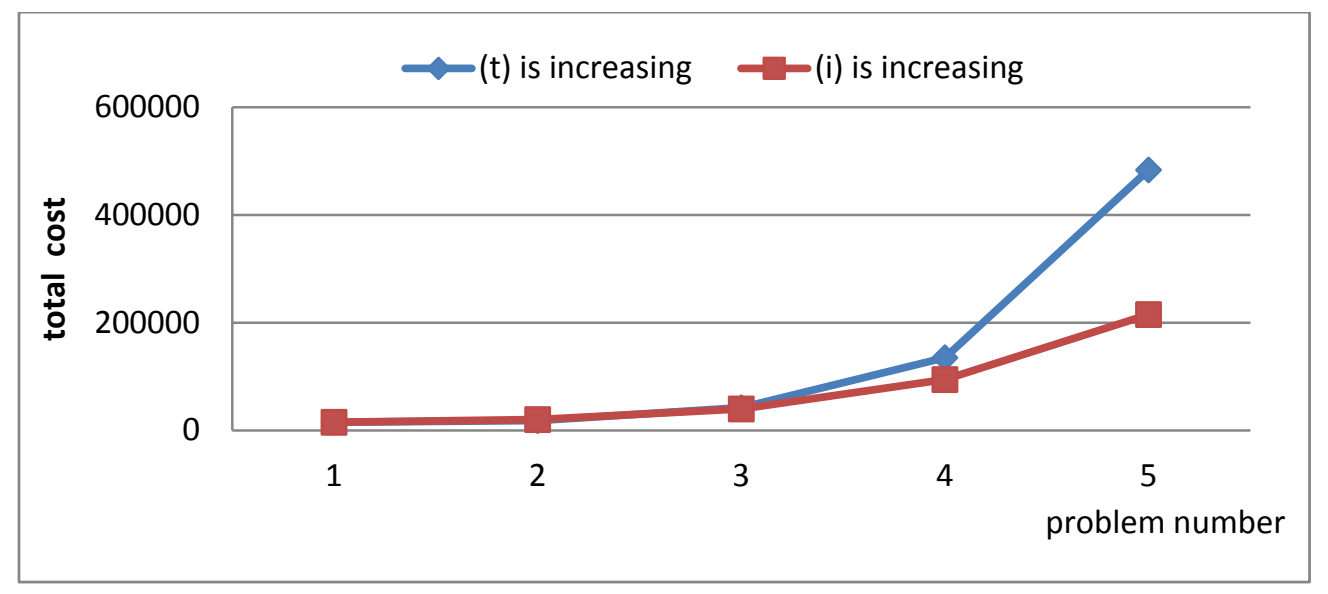

Fig. 5. Analysis of impact of parameters $i$ and $t$ on the total cost 
Table 2

Analysis of the effect of candidate locations

\begin{tabular}{|c|c|c|c|c|c|c|c|c|}
\hline$\dot{0}$ & $\begin{array}{l}\text { Problem } \\
\mathrm{I} \times \mathrm{K} \times \mathrm{t}\end{array}$ & 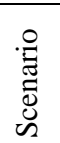 & Probability & Costs & Demands & $\begin{array}{l}\text { Total } \\
\text { costs }\end{array}$ & $\begin{array}{c}\text { Post-disaster } \\
\text { costs }\end{array}$ & $\begin{array}{c}\text { Holding } \\
\text { costs }\end{array}$ \\
\hline \multirow{4}{*}{1} & \multirow{4}{*}{$2 \times 2 \times 2$} & 1 & 0.4 & \multirow{4}{*}{ Unif [50-2000] } & \multirow{4}{*}{ Unif [1000-5000] } & \multirow{4}{*}{15293} & \multirow{4}{*}{5125} & \multirow{4}{*}{10168} \\
\hline & & 2 & 0.2 & & & & & \\
\hline & & 3 & 0.3 & & & & & \\
\hline & & 4 & 0.1 & & & & & \\
\hline \multirow{4}{*}{2} & \multirow{4}{*}{$2 \times 2 \times 4$} & 1 & 0.0 & \multirow{4}{*}{ Unif [50-2000] } & \multirow{4}{*}{ Unif [1000-5000] } & \multirow{4}{*}{20341} & \multirow{4}{*}{11325} & \multirow{4}{*}{9016} \\
\hline & & 2 & 0.2 & & & & & \\
\hline & & 3 & 0.3 & & & & & \\
\hline & & 4 & 0.1 & & & & & \\
\hline \multirow{4}{*}{3} & \multirow{4}{*}{$2 \times 2 \times 8$} & 1 & 0.4 & \multirow{4}{*}{ Unif [50-2000] } & \multirow{4}{*}{ Unif [1000-5000] } & \multirow{4}{*}{39810} & \multirow{4}{*}{20043} & \multirow{4}{*}{19767} \\
\hline & & 2 & 0.2 & & & & & \\
\hline & & 3 & 0.3 & & & & & \\
\hline & & 4 & 0.1 & & & & & \\
\hline \multirow{4}{*}{4} & & 1 & 0.4 & \multirow{4}{*}{ Unif [50-2000] } & \multirow{4}{*}{ Unif [1000-5000] } & \multirow{4}{*}{94620} & \multirow{4}{*}{66540} & \multirow{4}{*}{28080} \\
\hline & & 2 & 0.2 & & & & & \\
\hline & $2 \times 2 \times 10$ & 3 & 0.3 & & & & & \\
\hline & & 4 & 0.1 & & & & & \\
\hline \multirow{4}{*}{5} & \multirow{4}{*}{$2 \times 2 \times 32$} & 1 & 0.4 & \multirow{4}{*}{ Unif [50-2000] } & \multirow{4}{*}{ Unif [1000-5000] } & \multirow{4}{*}{215309} & \multirow{4}{*}{148741} & \multirow{4}{*}{66568} \\
\hline & & 2 & 0.2 & & & & & \\
\hline & & 3 & 0.3 & & & & & \\
\hline & & 4 & 0.1 & & & & & \\
\hline
\end{tabular}

\section{Numerical example for model II}

In this section, an inventory system is illustrated in order to verify model II in a fuzzy environment. In the considered system, we assume that total demand $\widetilde{D}$ and lead-time demand $\widetilde{d}$ are both described as triangular fuzzy numbers. The data are as follows. $\widetilde{D}=(3600,5300,5800), \widetilde{d}=(105,120,140), p=15$, $\pi=27, A=120$, and $h=15$. Through equations 29 and30, the obtained results are $Q^{*}=481.52$ and $r^{*}=141.08$; the range of $r$ is $[120,140]$, and $T \geq 3$. Furthermore, the sufficient condition,

$\frac{\partial^{2} \bar{M}(\widetilde{C}(Q, r))}{\partial Q^{2}} \frac{\partial^{2} \bar{M}(\widetilde{C}(Q, r))}{\partial r^{2}}-\frac{\partial^{2} \bar{M}(\widetilde{C}(Q, r))}{\partial Q \partial r}=0.702 \succ 0$, is satisfied. 
Since for triangular fuzzy numbers, for example $(A, B, C)$, there are three situations, which are $B-A>C$ $B, B-A=C-B$, and $B-A<C-B$, we run the model for all the nine situations, in order to demonstrate that the model is not only applicable for the designed example. Table 3 illustrates the results.

Table 3

The results of model II

\begin{tabular}{llll}
\hline$\widetilde{d}$ & $\widetilde{D}=(3600,4600,5600)$ & $\widetilde{D}=(3600,5300,5800)$ & $\widetilde{D}=(3600,4100,6000)$ \\
\hline & $\left(Q^{*}, r^{*}\right)$ & $\left(Q^{*}, r^{*}\right)$ & $\left(Q^{*}, r^{*}\right)$ \\
\hline$(60,75,110)$ & $(395.36,95.55)$ & $(405.51,102,77)$ & $(385.33,100.20)$ \\
$(60,100,110)$ & $(463.20,126.32)$ & $(416.00,118.29)$ & $(407.99,116.23)$ \\
$(45,55,65)$ & $(251.09,40.50)$ & $(293.42,63.02)$ & $(246.16,39.87)$ \\
\hline
\end{tabular}

\section{Conclusions and future research directions}

This paper has presented two mathematical models that optimize pre- and post-event planning for meeting demands of all the phases of disaster relief lifecycle. Perishable commodities holding costs, as mentioned in the literature, considerably affect the decisions made regarding locations of the storage facilities and their inventory levels. This paper, for the first time, has incorporated the perishable items' inventory policies into prepositioning decisions. In other words, considering perishable supplies holding costs, a two-stage stochastic programming model has been developed in which prepositioning locations, inventory allocating quantities, short-term distribution quantities, and replenishment schedules are determined. To ensure that the relief warehouses of affected areas are to be able to support relief operations until the last day of recovery phase, we need to propose a special inventory control model for the recovery and reconstruction phase of relief operations. However, although this period has a desperate need of inventory planning, there is no appropriate inventory model in the literature for it. To fulfill this gap, a continuous review $(Q, r)$ inventory model has been proposed in which the order quantities and re-order points are determined while considering uncertain demands. Due to the unique circumstances of long-term response phase, a mixture of backorder and lost demands has been considered so that the backorder demands are transformed to lost ones if the stock-out period lasts more than several days. For this model, a fuzzy ranking method by using the possibilistic mean operator is adopted to achieve the optimal solution. In order to illustrate the applicability of the proposed models, a case study and a numerical example have also been provided for the preparedness and recovery phases, respectively.

Given the difficulty of estimation of suitable values for shortage penalties, we ran model I for eleven times under different penalty cost values. The results demonstrated that the values of penalty costs should be neither too small nor too large, and for attaining the best value, a similar sensitive analysis should be performed. This research has proposed a continuous review $(Q, r)$ inventory model for the recovery phase of the disaster cycle for the first time. However, developing other inventory models, including FOI models, in this regard is of particular interest.

\section{References}

Altay, N., \& Green, W. G. (2006). OR/MS research in disaster operations management. European Journal of Operational Research, 175(1), 475-493.

Balcik, B., \& Beamon, B. M. (2008). Facility location in humanitarian relief. International Journal of Logistics, 11(2), 101-121.

Barbaroso\&gcaron, G. (2004). A two-stage stochastic programming framework for transportation planning in disaster response. Journal of the Operational Research Society, 55(1), 43-53. 
Beamon, B. M., \& Kotleba, S. A. (2006). Inventory modelling for complex emergencies in humanitarian relief operations. International Journal of Logistics: Research and Applications, 9(1), $1-18$.

Carlsson, C., \& Fullér, R. (2001). On possibilistic mean value and variance of fuzzy numbers. Fuzzy Sets and Systems, 122(2), 315-326.

Davis, L. B., Jiang, S. X., \& Terry, J. (2013, May). Empirical modeling of in-kind donations for a nonprofit hunger relief organization. In industry studies association annual conference.

Dutta, P., Chakraborty, D., \& Roy, A. R. (2007). Continuous review inventory model in mixed fuzzy and stochastic environment. Applied Mathematics and Computation, 188(1), 970-980.

Haghani, A., \& Oh, S. C. (1996). Formulation and solution of a multi-commodity, multi-modal network flow model for disaster relief operations. Transportation Research Part A: Policy and Practice, 30(3), 231-250.

Kovács, G., \& Spens, K. M. (2007). Humanitarian logistics in disaster relief operations. International Journal of Physical Distribution \& Logistics Management, 37(2), 99-114.

Ozbay, K., \& Ozguven, E. E. (2007). Stochastic humanitarian inventory control model for disaster planning. Transportation Research Record: Journal of the Transportation Research Board, 2022(1), 63-75.

Rath, S., \& Gutjahr, W. J. (2014). A math-heuristic for the warehouse location-routing problem in disaster relief. Computers \& Operations Research, 42, 25-39.

Rawls, C. G., \& Turnquist, M. A. (2010). Pre-positioning of emergency supplies for disaster response. Transportation research part B: Methodological, 44(4), 521-534.

Rawls, C. G., \& Turnquist, M. A. (2012). Pre-positioning and dynamic delivery planning for shortterm response following a natural disaster. Socio-Economic Planning Sciences, 46(1), 46-54.

Salas, L. C., Cárdenas, M. R., \& Zhang, M. (2012). Inventory policies for humanitarian aid during hurricanes. Socio-Economic Planning Sciences, 46(4), 272-280.

Salmerón, J., \& Apte, A. (2010). Stochastic optimization for natural disaster asset prepositioning. Production and Operations Management, 19(5), 561-574.

Thomas, A., \& Kopczak, L. R. (2007). Life-saving supply chains. In Building supply chain excellence in emerging economies (pp. 93-111). Springer US.

Tomasini, R. M., \& Van Wassenhove, L. (2009). Humanitarian logistics (pp. 44-47). Basingstoke: Palgrave Macmillan.

Tufinkgi, P. (2006). Logistik im kontext internationaler katastrophenhilfe: Entwicklung eines logistischen referenzmodells für katastrophenfälle. Haupt.

Ukkusuri, S. V., \& Yushimito, W. F. (2008). Location routing approach for the humanitarian prepositioning problem. Transportation Research Record: Journal of the Transportation Research Board, 2089(1), 18-25.

Wang, L., Fu, Q. L., \& Zeng, Y. R. (2012). Continuous review inventory models with a mixture of backorders and lost sales under fuzzy demand and different decision situations. Expert Systems with Applications, 39(4), 4181-4189.

Whybark, D. C. (2007). Issues in managing disaster relief inventories. International Journal of Production Economics, 108(1), 228-235. 


\section{Appendix}

The case study data

\begin{tabular}{|c|c|c|c|c|c|c|c|c|c|}
\hline County & Scenario & Probability & Demand & & County & Scenario & Probability & Demand & \\
\hline & & & $\begin{array}{c}\text { Commodity } \\
1\end{array}$ & $\begin{array}{c}\text { Commodity } \\
2\end{array}$ & & & & $\begin{array}{c}\text { Commodit } \\
\text { y } 1\end{array}$ & $\begin{array}{c}\text { Commodit } \\
\text { y } 2\end{array}$ \\
\hline \multirow{3}{*}{1} & 1 & .01 & 6300 & 4000 & \multirow{3}{*}{16} & 1 & .25 & 5100 & 5500 \\
\hline & 2 & .5 & 5000 & 4200 & & 2 & .56 & 6500 & 8400 \\
\hline & 3 & .07 & 6000 & 2700 & & 3 & .24 & 5200 & 5300 \\
\hline \multirow{3}{*}{2} & 1 & .09 & 5500 & 9000 & \multirow{3}{*}{17} & 1 & .55 & 3200 & 3100 \\
\hline & 2 & .55 & 10000 & 84000 & & 2 & .46 & 4500 & 4000 \\
\hline & 3 & .25 & 2400 & 3000 & & 3 & .43 & 7400 & 8000 \\
\hline \multirow{3}{*}{3} & 1 & .6 & 5200 & 3600 & \multirow{3}{*}{18} & 1 & .23 & 8400 & 8000 \\
\hline & 2 & .2 & 3500 & 5200 & & 2 & .12 & 3200 & 4200 \\
\hline & 3 & .65 & 4700 & 9800 & & 3 & .01 & 2900 & 3000 \\
\hline \multirow{3}{*}{4} & 1 & .36 & 7500 & 5600 & \multirow{3}{*}{19} & 1 & .09 & 5000 & 5200 \\
\hline & 2 & .2 & 5200 & 3800 & & 2 & .07 & 4900 & 4800 \\
\hline & 3 & .04 & 4400 & 3900 & & 3 & .06 & 8400 & 8600 \\
\hline \multirow{3}{*}{5} & 1 & .08 & 5800 & 9500 & \multirow{3}{*}{20} & 1 & .18 & 4000 & 4500 \\
\hline & 2 & .05 & 5400 & 5600 & & 2 & .09 & 8800 & 9000 \\
\hline & 3 & .4 & 4500 & 5400 & & 3 & .26 & 7400 & 7600 \\
\hline \multirow{3}{*}{6} & 1 & .6 & 6500 & 8800 & \multirow{3}{*}{21} & 1 & .26 & 5500 & 5600 \\
\hline & 2 & .7 & 8900 & 9200 & & 2 & .42 & 6500 & 6800 \\
\hline & 3 & .25 & 3600 & 6600 & & 3 & .23 & 8400 & 8500 \\
\hline \multirow{3}{*}{7} & 1 & .03 & 5400 & 3200 & \multirow{3}{*}{22} & 1 & .15 & 6500 & 6900 \\
\hline & 2 & .35 & 8700 & 7100 & & 2 & .01 & 9800 & 10000 \\
\hline & 3 & .46 & 2500 & 4500 & & 3 & .46 & 3200 & 3500 \\
\hline \multirow{3}{*}{8} & 1 & .25 & 9400 & 6200 & \multirow{3}{*}{23} & 1 & .08 & 4500 & 4100 \\
\hline & 2 & .48 & 7400 & 9500 & & 2 & .43 & 6500 & 6500 \\
\hline & 3 & .45 & 7500 & 9400 & & 3 & .13 & 3900 & 3600 \\
\hline \multirow{3}{*}{9} & 1 & .26 & 7500 & 6500 & \multirow{3}{*}{24} & 1 & .05 & 3700 & 4100 \\
\hline & 2 & .24 & 3800 & 3400 & & 2 & .13 & 8200 & 8000 \\
\hline & 3 & .55 & 9500 & 9200 & & 3 & .46 & 3900 & 4200 \\
\hline \multirow{3}{*}{10} & 1 & .65 & 6200 & 6500 & \multirow{3}{*}{25} & 1 & .06 & 8100 & 8000 \\
\hline & 2 & .08 & 5300 & 3500 & & 2 & .13 & 2900 & 3000 \\
\hline & 3 & .07 & 4100 & 8700 & & 3 & .04 & 4800 & 4500 \\
\hline \multirow{3}{*}{11} & 1 & .35 & 7200 & 6300 & & 1 & .23 & 8100 & 8000 \\
\hline & 2 & .02 & 3400 & 8500 & 26 & 2 & .46 & 8000 & 7900 \\
\hline & 3 & .23 & 6800 & 7400 & & 3 & .52 & 5100 & 5000 \\
\hline & 1 & .46 & 9500 & 8400 & & 1 & .23 & 6000 & 6200 \\
\hline 12 & 2 & .23 & 4500 & 6800 & 27 & 2 & .46 & 4000 & 4200 \\
\hline & 3 & .15 & 8400 & 8800 & & 3 & .26 & 5200 & 5300 \\
\hline & 1 & .46 & 9800 & 8300 & & 1 & .46 & 4000 & 4500 \\
\hline 13 & 2 & .55 & 8500 & 5200 & 28 & 2 & .13 & 8400 & 8400 \\
\hline & 3 & .46 & 4500 & 3500 & & 3 & .28 & 9500 & 9000 \\
\hline & 1 & .23 & 7100 & 6400 & & 1 & .23 & 4500 & 5000 \\
\hline 14 & 2 & .48 & 5200 & 5600 & 29 & 2 & .46 & 6500 & 6600 \\
\hline & 3 & .46 & 3400 & 5400 & & 3 & .56 & 5400 & 5200 \\
\hline & 1 & .23 & 3200 & 6200 & & 1 & .27 & 6500 & 6300 \\
\hline 15 & 2 & .05 & 8000 & 2400 & 30 & 2 & .46 & 4500 & 4200 \\
\hline & 3 & .06 & 4100 & 8400 & & 3 & .02 & 8000 & 8100 \\
\hline
\end{tabular}

